Enfin, le sérum extrait de la " Paracurd " est plus riche en ES qu'un sérum ordinaire, le volume étant moindre bien entendu.

C'est en définitive toute une révolution dans la "Fromagerie de Demain " qu'apportent les procédés Hutin :

Non seulement la perspective de fabrications continues avec toute l'automation désirable pour la production de fromages de qualité constante, mais également la perspective de spécialisation de certaines usines dans le ramassage, la concentration du lait et son expédition dans des usines fromagères; enfin la perspective de conditions d'exploitation très améliorées et, très probablement, celle du développement de produits fromagers nouveaux.

\title{
UN FROMAGE CONTAMINÉ PAR SHIGELLA SONNEI, CAUSE D'INTOXICATION ALIMENTAIRE
}

\author{
par
}

MM. SZTURM-RUBINSTEN (2), A.-L. COURTIEU (3) et G. MAKA (3)

Au mois de novembre 1963 la Sous-Direction de l'Hygiène Publique a été alertée par quelques cas de dysenterie bacillaire concernant deux familles de la Loire et une famille de la HauteLoire. Ces cas avaient une origine commune : la consommation de fromage du pays.

Les malades ont été hospitalisés à Saint-Etienne le 8 novembre 1963 et les examens bactériologiques ont été effectués dansle laboratoire de cet hôpital. Voici les détails que nous avons pu obtenir sur cette intoxication alimentaire : six membres de deux familles ont présenté un syndrome diarrhée-vomissements-fièvreétat de collapsus; sur cinq coprocultures pratiquées (sur les milieux SS et gélose lactosée au bromocrésol pourpre), Shigella sonnei a été isolée deux fois : le 8 novembre $1963 \mathrm{chez}$ un enfant dans une famille, le 9 novembre 1963 chez un adulte dans l'autre.

Les aliments incriminés ont été tout d'abord les conserves. La recherche des germes pathogènes dans ces produits a été négative, aucune Salmonella n'a été décelée. L'autre aliment consommé par les deux familles est un fromage de vache du pays, frais, couvert d'une mince pellicule de moisissure et acheté chez le même fournisseur.

(1) Bull. Acad. Med., 1964, [148], 480.

(2) C.N.R.S. et Institut Pasteur, Centre National des Shigella.

(3) Centre hospitalier de Saint-Etienne. 
De chacun des deux échantillons de fromage saisis par la Brigade de Gendarmerie de Firminy, chez les deux familles, une souche de Sh. sonnei a été isolée le 9 novembre 1963.

Le 14 novembre 1963, six jours après l'hospitalisation des malades, un autre cas d'intoxication alimentaire chez un enfant est signalé dans la Haute-Loire. Sh. sonnei est isolée par coproculture. La petite malade serait apparentée au fournisseur déjà mentionné et consommait du lait ou du fromage provenant de sa ferme.

Le même jour, la direction de la Santé de la Loire, a saisi sur le marché de Firminy, le fromage sec et gras vendu par le fermier. La recherche des Shigella a été négative.

A la même date, des écouvillonnages rectaux sont pratiqués toujours par la direction de la Santé de la Haute-Loire, pourla femme du cultivateur et ses trois enfants âgés de 4 à 7 ans. A noter qu'aucun prélèvement n'a été fait chez le fermier. Les prélèvements ont été reçus par le laboratoire quarante-huit heures plus tard, aucune Shigella n'a été trouvée. L'examen, répété après huit jours, a donné également un résultat négatif. Au même moment les Shigella ont été recherchées dans la ferme : dans trois échantillons d'eau de puits, dans un échantillon de lait de vache, de lait de chèvre, fromage de chèvre et de vache, partout sans succès.

Les souches de $\$$ h. sonnei isolées par coproculture des trois malades et celles isolées des échantillons du fromage ont été adressées au Centre National des Shigella pour détermination du biotype et du lysotype. Elles sont toutes les cinq identiques et appartiennent au biotype " a " et au lysotype " 2 ". Ces biotypes et lysotypes ne sont pas très fréquents dans la région. On peut conclure, tout au moins pour deux malades, que la source de contamination était la même : le fromage lui-même très vraisemblablement contaminé par la personne qui l'avait préparé. Il est évidemment regrettable que les écouvillonnages rectaux n'aient pas donné de résultats positifs : il n'a pas été possible d'établir avec certitude l'identité du porteur de germes. Les examens bactériologiques n'ont pu être faits d'une façon complète et satisfaisante dans la famille suspectée. Le fermier a échappé au prélèvement et les écouvillons provenant des autres sujets sont parvenus au laboratoire après un délai de quarante-huit heures : le retard apporté à l'ensemencement est peu favorable à la recherche des Shigella.

Il est de toute façon important du point de vue épidémiologique, de constater la présence peut-être durable de Shigella sonnei dans le fromage. L'un de nous [4] a observé une contamination par une glace alimentaire : la souche de Sh. sonnei de cette glace et celles obtenues par coprocultures des personnes qui l'avaient consommée, appartenaient à un même biotype et à un même lysotype.

Ces deux observations confirment l'intérêt qu'a pour l'épidémiologie la détermination du biotype et du lysotype de Shigella sonnei. 
La survie des Shigella dans les produits alimentaires a déjà été étudiée par un certain nombre d'auteurs.

Savoiskii [2], dans les conserves de légumes, a trouvé que $S h$. sonnei est plus longtemps repiquable que $S h$. dys. 1 et $S h$. flexneri.

Steuer [3] a étudié la survie de $S h . d y s .1, S h$. flexneri et $S h$. sonnei dans le lait et le beurre contaminés expérimentalement et gardés à des températures et dans des conditions d'acidité variables. C'est $\$$ h. sonnei qui s'est avérée la plus résistante et qui avait les plus grandes possibilités de survivre dans ces aliments.

Nevot [1] avait aussi remarqué que Sh. sonnei présente une résistance à la chaleur plus grande que Sh. flexneri et Sh. dys. 1 .

Nous avons tenu à rapporter cette observation parce que la possibilité d'une contamination alimentaire par une Shigella est rarement envisagée. Bien que ce soit certainement moins fréquent qu'une intoxication due aux Salmonella ou aux staphylocoques, il faut tenir compte de cette éventualité, et penser d'abord à Sh. sonnei, bacille dysentérique le plus fréquemment isolé en France.

L'étude de la survie de $S h$. sonnei dans un fromage du même genre que celui qui a été contaminé est en cours. On peut, d'ores et déjà, affirmer que dans ce fromage gardé à +40 , contaminé même avec une petite quantité de germes, la survie peut être de l'ordre de deux mois.

\section{Résumé}

Trois personnes, après avoir consommé du fromage de vache frais, et vendu par un même fournisseur, ont présenté une intoxication alimentaire. Des selles des malades ainsi que de deux échantillons de fromage, une souche de Shigella sonnei a été isolée, du même biotype et du même lysotype. Il est évident que c'est le fromage qui a été la source de contamination. Sh. sonnei est le bacille dysentérique le plus fréquent en France; il survit aussi plus longtemps et plus facilement que les autres Shigella dans les produits alimentaires.

\section{BIBLIOGRAPHIE}

[1] A. Nevot, P. Lafont et J. Lafont, Ann. Inst. Pasteur, 1960, [98], 306.

[2] I. I. SAvorskrI. Tr. Kharkovski Med. Inst,, 1958, [40], 92.

[3] W. Steukr. Arch. Hyg., 1941, [120], 233.

[4] S. Szturm-Rubinsten, R. Chamfeuil, M. Huet et J. Manawtaud. Ann. Inst. Pasteur, [99], 456.

[5] S. Szturm-Rubinsten, Ann. Inst. Pasteur, [106], 14. 